\title{
Magnetic field decay in black widow pulsars
}

\author{
Camile Mendes, ${ }^{1 \star}$ Marcio G. B. de Avellar, ${ }^{1 \star}$ J. E. Horvath, ${ }^{1 \star}$ Rodrigo A. de Souza,${ }^{1}$ \\ O. G. Benvenuto ${ }^{2,3}$ and M. A. De Vito Vi,3 $^{2,3}$ \\ ${ }^{1}$ Instituto de Astronomia, Geofísica e de Ciências Atmosféricas, Universidade de São Paulo, Rua do Matão 1226, 05508-090 São Paulo, Brasil \\ ${ }^{2}$ Instituto de Astrofísica de La Plata, IALP, B1900FWA CCT-CONICET-UNLP, Argentina \\ ${ }^{3}$ Facultad de Ciencias Astronómicas y Geofísicas, Universidad Nacional de La Plata (UNLP), Paseo del Bosque S/N, B1900FWA La Plata, Argentina
}

Accepted 2017 December 18. Received 2017 November 21; in original form 2017 August 21

\begin{abstract}
We study in this work the evolution of the magnetic field in 'redback-black widow' pulsars. Evolutionary calculations of these 'spider' systems suggest that first the accretion operates in the redback stage, and later the companion star ablates matter due to winds from the recycled pulsar. It is generally believed that mass accretion by the pulsar results in a rapid decay of the magnetic field when compared to the rate of an isolated neutron star. We study the evolution of the magnetic field in black widow pulsars by solving numerically the induction equation using the modified Crank-Nicolson method with intermittent episodes of mass accretion on to the neutron star. Our results show that the magnetic field does not fall below a minimum value ('bottom field') in spite of the long evolution time of the black widow systems, extending the previous conclusions for much younger low-mass X-ray binary systems. We find that in this scenario, the magnetic field decay is dominated by the accretion rate, and that the existence of a bottom field is likely related to the fact that the surface temperature of the pulsar does not decay as predicted by the current cooling models. We also observe that the impurity of the pulsar crust is not a dominant factor in the decay of magnetic field for the long evolution time of black widow systems.
\end{abstract}

Key words: accretion, accretion discs-magnetic field-stars: neutron.

\section{INTRODUCTION}

There is an intense observational activity around the relativistic binary systems collectively known as 'spiders'. The number of these binary systems has recently increased due to the contributions of Fermi-LAT mission (Saz Parkinson, Belfiore \& Razzano 2014). The construction of a class of theoretical models of stellar evolution (Benvenuto, De Vito \& Horvath 2012) suggests that a small fraction of the relativistic binary systems evolves into configurations called 'redbacks' and later to 'black widows'. These systems have been tentatively identified as belonging to different phases of the same relativistic binary system, where X-ray illumination on to the donor star (redback stage) and the subsequent ablation of the donor's mass by the pulsar's wind (black widow stage) are unique elements and determine the evolution (Benvenuto, De Vito \& Horvath 2014).

It is generally believed that the accretion of mass by the pulsar, such as the one that occurs in the redback stage, results in a faster magnetic field decay than the magnetic field decay of an isolated neutron star (Shibazaki et al. 1989; Zhang 2013). However, despite

^E-mail: jbcamile@gmail.com (CM); mgb.avellar@iag.usp.br (MGBdeA); foton@iag.usp.br (JEH) the long evolution of the black widow systems, the magnetic field seems not to decay below a bottom field value, $B \sim 2 \times 10^{8} \mathrm{G}$, (Wang et al. 2011). The physical properties of the crust, where the magnetic field is anchored, and the influence of the accretion on to the compact star are the main ingredients thought to be involved in the explanation of the bottom field occurrence (Zhang \& Kojima 2006).

From simple and well-known evolutionary considerations (van den Heuvel 2011), we know that the time necessary to start the mass transfer in a binary system with a pulsar is at least $10^{9} \mathrm{yr}$ from the formation of the latter (here the companion is assumed to be a solartype star), and the posterior trajectory of the system follows from its initial position in the companion mass-orbital period plane (De Vito \& Benvenuto 2010). At the moment the companion star leaves the main sequence, the radius starts to increase until it fills the Roche Lobe of the system, and at this point, accretion of matter from the companion on to the compact star begins. For a period about $10^{9}$ $2 \times 10^{9} \mathrm{yr}$, this accretion occurs steadily (provided the donor mass is not too high). Since the mass accreted by the pulsar causes the production of X-rays, the phenomenon called feedback irradiation is important for tight binary systems (Benvenuto et al. 2014).

At some point along the evolution of the system, when the orbital period is $\sim$ hours, the irradiation destabilizes and destroys 
(partially) the accretion disc, stopping the accretion until the disc forms again. Observations of this intermittency show that this behaviour has a typical time-scale of few years and occurs within an interval of $2-4 \times 10^{9} \mathrm{yr}$ until the companion star is unable to transfer mass to the pulsar and the latter becomes again effectively isolated (Benvenuto et al. 2012). Although no measurements of the magnetic field at this intermittent stage exist, the accretion must cause some decay of the field from its original value. The mechanisms that operate for the field decay in compact objects have been studied over the years (Geppert \& Urpin 1994; Konar 1997; Cumming 2002; Payne \& Melatos 2004; Konar 2017). The evolution of the pulsar's magnetic field is driven mainly by the conductivity that strongly depends upon the impurity ratio in the crust of the compact star, a number believed to be fairly low before accretion begins and increases as a function of the accreted mass.

Another important factor in determining the conductivity is the temperature of the pulsar. Pulsars are born at very high temperatures (above $10^{9} \mathrm{~K}$ ), but they rapidly cool via neutrino emission down to $\sim 10^{6} \mathrm{~K}$. When accretion begins, the pulsar heats up again, and its actual temperature is a function of the accretion rate.

In this paper, we make a first study of the evolution of magnetic fields in 'spiders', which may be $\sim 100 \times$ older than other types of X-ray binaries (Benvenuto et al. 2012). We put on a firmer basis that it is the temperature, not the impurities, the factor that prevents the magnetic field to decay below the bottom field value due to a heating process inside the compact star. The many complexities of crust physics leading to the evolution of magnetic field have been discussed and explicated before (Elfritz et al. 2016), and for these reasons we consider our work to be indicative of the accurate very long-term behaviour.

In Section 2, we set the basic equations and the formalism, dedicating some space to discuss the Hall effect. In Section 3, we present the results of our analysis, and we discuss and present our conclusions of this study in Section 3.1.

\section{BASIC EQUATIONS AND FORMALISM}

The evolution of the binary systems that we intend to study can be roughly divided into three different phases (Benvenuto et al. 2014).

In the first phase the young pulsar is effectively isolated, since the companion star takes about $10^{9} \mathrm{yr}$ to leave the main sequence, fill the Roche lobe, and start the accretion. In this phase, the evolution of magnetic field is governed by the Ohmic diffusion and the induction equation given by

$$
\frac{\partial \boldsymbol{B}}{\partial t}=-\frac{c^{2}}{4 \pi} \nabla \times\left(\frac{1}{\sigma} \nabla \times \boldsymbol{B}\right),
$$

where $\sigma$ is the conductivity.

The second phase begins when the accretion of matter from the companion to the pulsar begins. This phase lasts for about another $\sim 10^{9} \mathrm{yr}$, assuming a constant (average) accretion rate. For this phase, we have to add a term representing the accretion to the induction equation.

$$
\frac{\partial \boldsymbol{B}}{\partial t}=\boldsymbol{\nabla} \times\left(\boldsymbol{v}_{e} \times \boldsymbol{B}\right)-\frac{c^{2}}{4 \pi} \nabla \times\left(\frac{1}{\sigma} \nabla \times \boldsymbol{B}\right),
$$

where $\boldsymbol{v}_{e}$ is the average local velocity of the fluid.

The third phase is characterized by the ablation phenomenon, which increases its importance as the stars become spatially closer. Fortunately, only the companion star is heavily affected but not the pulsar itself.

\subsection{Mass flow and matter transport}

As discussed in Konar (1997), the crustal mass of the pulsar remains effectively constant if the accretion on to the neutron star crust amounts up to $0.1 \mathrm{M}_{\odot}$. This implies that the density profile of the crust does not change appreciably. Assuming then that the mass flow on to the crust of the neutron star is spherically symmetric, our assumptions allow us to write.

$\dot{M} \mathrm{~d} t=4 \pi r^{2} \rho(r) \mathrm{d} r$,

where $\rho(\mathrm{r})$ is the density of the pulsar crust as a function of the radius $r$ of the star.

Because the flux of accreted material is radially inwards by hypothesis, the velocity $\boldsymbol{v}_{e}$ of the fluid is given by

$\boldsymbol{v}_{e}(r)=-\frac{\dot{M}}{4 \pi r^{2} \rho(r)} \hat{r}$,

where $\dot{M}$ is the mass accretion rate, which we assume to be a constant whenever accretion enters in scene.

\subsection{Density profile}

As is widely accepted, the neutron star features three different regions, basically: an outer crust with densities up to $4 \times 10^{11} \mathrm{~g} \mathrm{~cm}^{-3}$ (neutron drip density), an inner crust with densities between the neutron drip density and the nuclear density $\left(2.8 \times 10^{14} \mathrm{~g} \mathrm{~cm}^{-3}\right)$, and the core where we have a 'nucleon soup' of unknown detailed composition (Glendenning 1996; Becker 2009).

In this work, we assume for definiteness the Baym, Phetick and Sutherland (BPS) EoS (Baym, Pethick \& Sutherland 1971) for the outer crust, Negele and Vautherin (NV) EoS (Negele \& Vautherin 1973) for the inner crust, and Wiringa, Fiks and Fabrocini (WFF) EoS (Wiringa, Fiks \& Fabrocini 1988) for the core region. Other choices are possible but the final effects on the field history are expected to be minimal. We proceeded to obtain the density profile of the star, $\rho(r)$, by solving the Tolman-Oppenheimer-Volkoff (TOV) equations.

In this work, we consider that the magnetic field is anchored in the crust of neutron star and does not reach the core. Even if it seems an important restriction, we should remark that the magnetic field decay physics is not sensitive to the unknown exact composition of the core (García, Aguilera \& Romero 2014).

\subsection{Conductivity and temperature}

The outer crust of the neutron star can be in liquid or crystalline solid state depending on the local temperature (Slattery, Doolen \& Dewitt 1982).

The accretion on to the compact star stabilizes within a few thousand years (Savonije 1978) when the temperature achieves a steady value proportional to the accretion rate (Miralda-Escude, Paczynski \& Haensel 1990). The relation between the accretion rate, $\dot{M}$, and the crustal temperature, $T$, for accretion rates in the range $10^{-15} \mathrm{M}_{\odot} \mathrm{yr}^{-1}<\dot{M}<10^{-10} \mathrm{M}_{\odot} \mathrm{yr}^{-1}$ is given by (Zdunik et al. 1992)

$\log T=0.397 \log \dot{M}+12.35$,

where $T$ is in Kelvin and $\dot{M}$ is in $\mathrm{M}_{\odot} \mathrm{yr}^{-1}$. 
The state of the crust can be inferred just comparing its temperature, due to accretion, with the melting temperature of the material (Slattery et al. 1982):

$T_{\mathrm{m}}=0.2269 \times 10^{8} \frac{Z^{2}\left(\frac{\rho_{6}}{A}\right)^{1 / 3}}{171} \mathrm{~K}$,

where $\rho_{6}$ is the density in units of $10^{6} \mathrm{~g} \mathrm{~cm}^{-3}, Z$ is the atomic number, and $A$ is the mass number.

For the region in which the crust temperature, $T$, is higher than melting temperature, $T_{\mathrm{m}}$, the crust will be in liquid state and the conductivity is given by (Itoh et al. 1984)

$\sigma_{\text {liq }}=8.53 \times 10^{21} \frac{y^{3}}{Z \Lambda_{\text {Coulomb }}\left(1+y^{2}\right)}$,

where $y=\left(\frac{Z \rho_{6}}{A}\right)^{1 / 3}$.

For deeper regions of the star in which the temperature is lower than the local melting temperature, the conductivity is given by

$$
\begin{aligned}
\sigma_{\text {solid }}= & \frac{1}{\sigma_{\text {phonon }}^{-1}+\sigma_{\text {impurity }}^{-1}}, \\
& \left\{\begin{array}{l}
\sigma_{\text {impurity }}=8.53 \times 10^{21} x Z / Q \\
\sigma_{\text {phonon }}=1.24 \times 10^{20} \frac{x^{4}}{u T_{8}} \frac{\left(u^{2}+0.0174\right)^{1 / 2}}{\left(1+1.1018 x^{2}\right) I_{\sigma}}
\end{array},\right.
\end{aligned}
$$

where

(i) $x \equiv \frac{r}{10.351[\mathrm{~km}]}$ is the dimensionless radius (the scale is related to the way we solved the TOV equations),

(ii) $\mathrm{Q}$ is the impurity parameter,

(iii) $u=\frac{2 \pi}{9}(\log \rho-3)$,

(iv) $T_{8}$ is the temperature in units of $10^{8} \mathrm{~K}$,

(v) $I_{\sigma}$ is a function of density, $Z$, and $A$.

We now have all the basic necessary elements to evolve the magnetic field according to the induction equation.

\subsection{The hall effect and the geometry of the magnetic field}

Initially, we consider a dipolar magnetic field anchored in the crust of the neutrons star, but the evolution of the temperature in the neutron star crust leads to a change of the geometry of the magnetic field (Viganò et al. 2015). In fact, for a magnetic field satisfying the Force-Free model (in $\nabla \times \boldsymbol{B}=\mu \boldsymbol{B}$ e $\boldsymbol{B} \cdot \nabla \mu=0$ is satisfied), the effect of the resistivity gradient modifies the geometry of the magnetic field from dipolar to a field with poloidal and toroidal components. This change in the geometry of the magnetic field brings up another effect on the magnetic field, the Hall effect. As in the case of the Ohmic diffusion, the Hall effect also leads to magnetic field decay (Aguilera, Pons \& Miralles 2008).

Considering the first evolution phase of the binary system in which accretion did not start yet, the equation for the evolution of the magnetic field is given by just (Aguilera et al. 2008)

$$
\frac{\partial \boldsymbol{B}}{\partial t}=-\frac{c}{4 \pi e} \nabla \times\left(\frac{\nabla \times \boldsymbol{B}}{n_{\mathrm{e}}} \times \boldsymbol{B}\right)+\frac{c^{2}}{4 \pi} \nabla \times\left(\frac{1}{\sigma} \nabla \times \boldsymbol{B}\right),
$$

where the first term corresponds to the Hall effect, $n_{\mathrm{e}}$ is the electron density, $e$ is the electron charge, and the second term corresponds to Ohmic diffusion.

By looking at the Hall and Ohmic time-scales implicit in these terms, we can divide the evolution of the magnetic field in two different stages: a first stage of rapid (but not exponential) decay of the field and a second stage driven by purely Ohmic dissipation

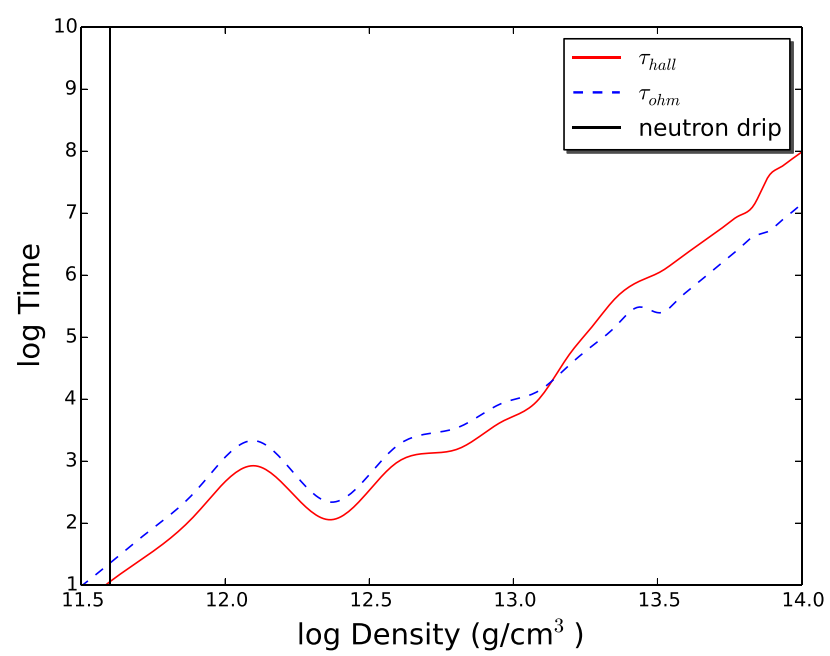

Figure 1. Comparison between $\tau_{\mathrm{ohm}}$ and $\tau_{\text {hall }}$. The vertical line is the neutron drip density.

(exponential). Therefore, with the time-scale of the Ohmic decay $\left(\tau_{\mathrm{ohm}}=\frac{4 \pi \sigma H^{2}}{c^{2}}\right)$ and the time-scale of the Hall effect $\left(\tau_{\text {hall }}=\frac{4 \pi e n_{\mathrm{e}}}{c B_{0} \mu^{2}}\right)$ (Cumming, Arras \& Zweibel 2004), we can cast the induction equation as (Igoshev \& Popov 2015, see)

$\frac{\mathrm{d} B}{\mathrm{~d} t}=-\frac{B}{\tau_{\mathrm{ohm}}}-\frac{1}{B_{0}} \frac{B^{2}}{\tau_{\text {hall }}}$,

whose simplest solution is given by

$B=B_{0} \frac{\exp \left(-t / \tau_{\mathrm{ohm}}\right)}{1+\frac{\tau_{\mathrm{ohm}}}{\tau_{\text {hall }}}\left(1-\exp \left(-t / \tau_{\mathrm{ohm}}\right)\right)}$.

In this case, we have

$t \ll \tau_{\text {ohm }} \rightarrow B \simeq B_{0} \frac{1}{1+t / \tau_{\text {hall }}}$

$t \geq \tau_{\mathrm{ohm}} \rightarrow B \simeq B_{0} \frac{\exp \left(-t / \tau_{\mathrm{ohm}}\right)}{1+\tau_{\mathrm{ohm}} / \tau_{\mathrm{hall}}}$.

In order to estimate and compare $\tau_{\text {ohm }}$ and $\tau_{\text {hall }}$, we assumed an initial $B_{0}=10^{13} G$, an value for the electron density given by $n_{\mathrm{e}}=\frac{Z}{A} n_{\mathrm{b}}\left(n_{\mathrm{b}}\right.$ is the baryonic density) and the pressure scaleheight $H=77.6 m \rho_{12}^{1 / 3}\left(Y_{e} / 0.25\right)^{4 / 3}\left(2.45 / g_{14}\right)$ (Cumming et al. 2004).

We can graphically appreciate the comparison between $\tau_{\text {ohm }}$ and $\tau_{\text {hall }}$ in Fig. 1. From Fig. 1, we see that the time-scales for Ohmic and Hall decay are of the same order, which implies that one could not, in principle, neglect completely the Hall effect.

However, in Fig. 2, we show that the magnetic field decay due to the Hall effect plus the Ohmic diffusion is similar to the magnetic field decay due to the Ohmic diffusion only, allowing us to neglect the Hall effect in our calculations, which, in turn, allows us to assume a purely dipolar geometry for the magnetic field (Konar 1997; Sengupta 1998; García et al. 2014) as a simplifying case.

\subsection{Numerical setup}

To solve the equation (2), we introduce a vector potential $\boldsymbol{A}$ such that $\boldsymbol{B}(r, \theta, \phi)=\nabla \times \boldsymbol{A}$, where $\boldsymbol{A}=\left(0,0, A_{\phi}\right)$, in spherical coordinates.

Assuming $A_{\phi}=\frac{g(r, t) \sin \theta}{r}$, where $g(r, t)$ is the Stokes' function, we are guaranteed to maintain a dipolar geometry for the field. 


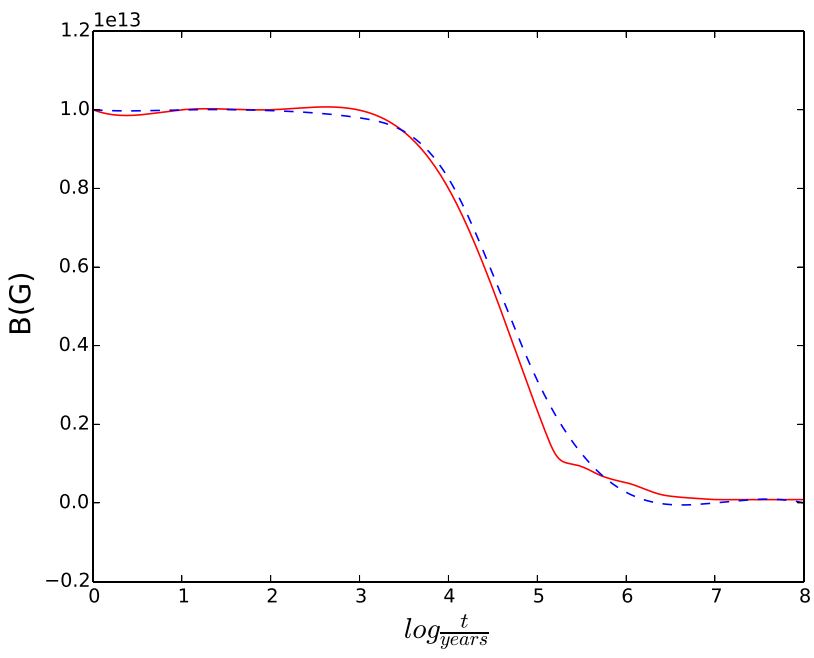

Figure 2. Comparison between the result, the decay of the magnetic field for a dipolar configuration (blue line), and a poloidal+toroidal configuration (red line).

Writing the induction equation (2) in terms of the dimensionless radial coordinate, we end up with the following induction equation, which we solved numerically:

$\frac{\partial g(x, t)}{\partial t}=V(x) \frac{\partial g(x, t)}{\partial x}+S(x, t)\left(\frac{\partial^{2} g(x, t)}{\partial x^{2}}-\frac{2 g(x, t)}{x^{2}}\right)$,

where $V(x)=V(r) / R$ and $S(x, t)=c^{2} /\left[4 \pi \sigma(r, t) R^{2}\right]$.

The boundary conditions are (Geppert \& Urpin 1994)

$\left.\frac{\partial g(x, t)}{\partial x}\right|_{x=X}+\frac{g(X, t)}{X}=0$

$g\left(x_{c o}, t\right)=0$,

where $X=1.0769$ is the dimensionless stellar radius and $x_{c o}$ is that radius to which the original boundary between the core and the crust is pushed to, due to accretion, at any point of time.

The initial distribution of the magnetic field is given by $g(x$, $0)=1-\left(x / x_{0}\right)^{2}$, where $x_{0}$ is the maximal depth the field penetrates the crust (see Geppert \& Urpin 1994). We fixed throughout the paper $x_{0}=1.039$.

As we assumed a magnetic field anchored in the crust of the neutron star, we assume that the conductivity between the crust and the core is $\sigma \approx 10^{50} \mathrm{~s}^{-1}$ to simulate the infinite conductivity in this homogeneous core region.

We modelled the neutron star as a TOV star with $R=11.15 \mathrm{~km}$, $M=1.33 \mathrm{M}_{\odot}$ and total crustal mass $M=0.039 \mathrm{M}_{\odot}$. We assume this value for mass knowing that with accretion the original crust is pushed on to the nucleus. Since we do not have a significant change in the density profile during this process, we assumed a constant density profile throughout the evolution of the star.

For the first phase (without accretion), we employed an initial temperature $10^{9} \mathrm{~K}$ (a typical value for the NS temperature $\sim$ hours after its birth). For the second phase, the temperature is mainly determined by the accretion. With an average accretion rate of $\dot{M}=10^{-10} \mathrm{M}_{\odot} \mathrm{yr}^{-1}$, the temperature is estimated to be $T=10^{8} \mathrm{~K}$. For the third phase, we use an initial temperature $10^{8} \mathrm{~K}$, resulting in a final value $10^{6} \mathrm{~K}$ approximately (see below). We did not incorporate the coupled thermal evolution in our scheme, but rather worked with the temperature as an external parameter to be specified at any time.

Analogously, we have considered three values for impurities, one for each stage. In the first phase, we have assumed a crust with a low impurity content $(Q=10)$. In the accretion phase, this number increases to about $Q=100$. In the last phase, after accretion stops, the crust recovers itself quickly decreasing somewhat the value of the impurities (to $Q \sim 40$, see below) (Roggero \& Reddy 2016).

We solved equation (10) using the modified Crank-Nicolson scheme, which is stable if the Courant condition given by $\frac{\delta t}{\delta x} V(x) \leqslant 1$ is satisfied for all the points of the grid and all the time.

In the modified Crank-Nicolson method, we combined the upwind method with the Crank-Nicolson method. The upwind method is used in the advection of the magnetic field and the CrankNicolson method is used in the diffusive part of the magnetic field. The Crank-Nicolson method is unconditionally stable for any size of the temporal or spatial grid but the upwind method is stable if the Courant condition is satisfied.

Knowing the values of $V(x)$ for a given accretion rate and the depth in the neutron star crust that the magnetic field is anchored, we make a combination between the values for the time-step $\delta t$ and the space grid. We find that a combination of the time interval $\delta t=10^{-3} \mathrm{yr}$ and a space grid of 83 points satisfies Courant condition for all the points of the grid and all the time.

\section{RESULTS}

We present now the results of our analysis on the evolution of the magnetic field of the pulsar in a redback/black widow binary system. These results can be seen in Fig. 3.

In the first phase of the evolution, the pulsar and the companion star are not interacting, thus the magnetic field decays by pure Ohmic diffusion. This is shown in the first part of Fig. 3, e.g. in the first $2 \mathrm{Gyr}$, the estimated duration of this phase. Considering an initial magnetic field $B_{0}\left(=10^{13} \mathrm{G}\right.$ here $)$, it remains fairly constant for the first $10^{4} \mathrm{yr}$, but decays sharply and continuously for the next $10^{5} \mathrm{yr}$ by about 2 orders of magnitude. From this point on and for the next $\sim 2 \times 10^{9} \mathrm{yr}$, the field remains essentially constant.

In the second part of Fig. 3, e.g. from $\sim 2 \times 10^{9}$ to $\sim 4 \times 10^{9} \mathrm{yr}$, between the black vertical lines, we show the magnetic field evolution in the second phase, where the accretion begins and becomes intermittent. As expected, the accretion makes the field to decay faster.

In this phase, lasting $\simeq 2 \times 10^{9} \mathrm{yr}$, the initial magnetic field decays from $\simeq 10^{-2.07} \mathrm{~B}_{0}$ to $\simeq 10^{-2.5} \mathrm{~B}_{0}$, This decay occurs irregularly due to the intermittent accretion, although our values refer to an effective continuous process proceeding with an average mass accretion rate, as described above.

In the third stage of evolution, the accretion is negligible again and the magnetic field decays solely due to Ohmic diffusion until an age of $10 \mathrm{Gyr}$ (the age inferred for the black widow pulsars in some scenarios, see, for example, Benvenuto et al. 2012, 2014).

In the third part of Fig. 3, we show the decay of the magnetic field in the third phase of evolution. The initial magnetic field in this phase is approximately $10^{-2.5} \mathrm{~B}_{0}$. For a period of approximately $1.5 \times 10^{9} \mathrm{yr}$, the field decays is negligible, thereafter the magnetic field decays by approximately 2 orders of magnitude down to $10^{-4.5} \mathrm{~B}_{0}$ in $2 \times 10^{9} \mathrm{yr}$, when the system is $\sim 8 \times 10^{9} \mathrm{yr}$ old. From this point on, it is not possible to observe the decay of the field and we state that the magnetic field reached the 'bottom field' value. 


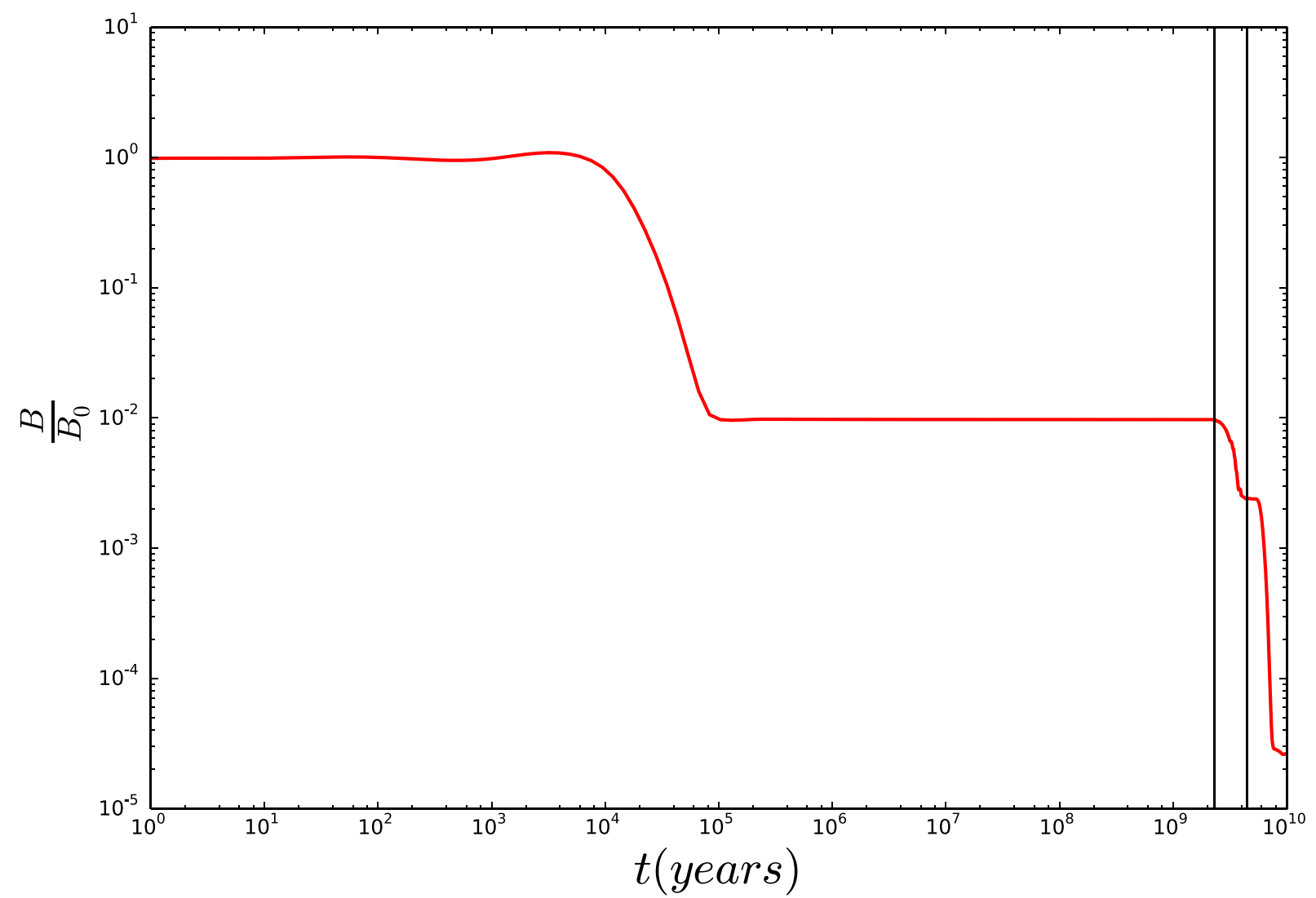

Figure 3. Evolution of the magnetic field in the pulsar for $10 \mathrm{Gyr}$. We calculated the evolution in three phases: In the first phase, there is no accretion and the field decays for $2 \times 10^{9} \mathrm{yr}$ solely via Ohmic decay. The second phase comprised between the two vertical black lines, the accretion process begins and become intermittent. The second phase lasts roughly $2 \times 10^{9} \mathrm{yr}$. The third phase, again there is no accretion and the field decays via Ohmic decay until $10 \times 10^{9} \mathrm{yr}$.

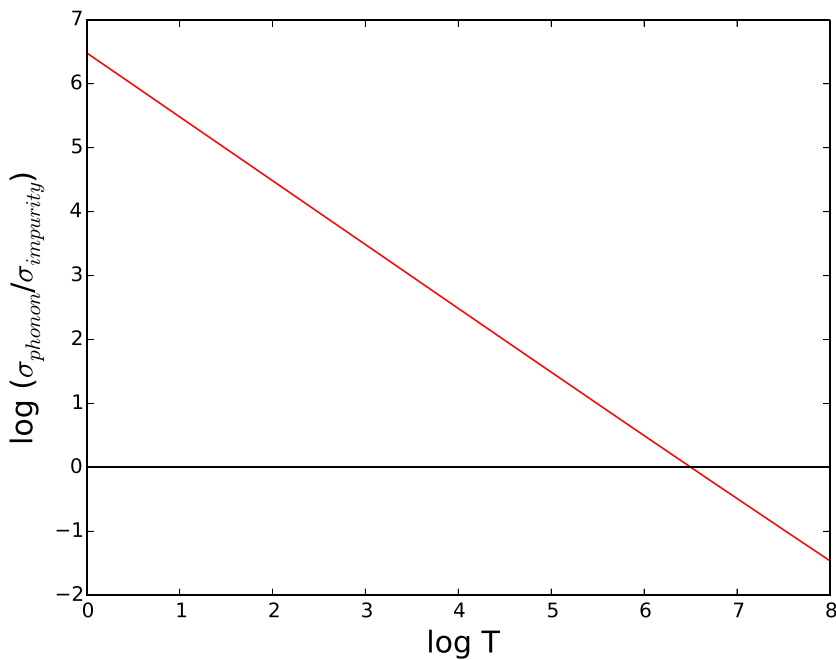

Figure 4. The combined phonon+impurity conductivity versus the temperature of the pulsar.

\subsection{The role of impurities}

It is important to assess how the increase of the impurities affects the magnetic field decay. To understand this problem, we performed an analysis of the conductivity of the pulsar crust due the combined effects of phonons and impurities. We present the result in Fig. 4.

As we see in equation (8), the conductivity due the phonons depends of the temperature as $1 / T$, and from Fig. 4 , we see that for temperatures above the $\simeq 10^{6.5} \mathrm{~K}$ the conductivity due the impurities is higher than the conductivity due the phonons. Therefore, in the (second) accretion phase where the temperature is approximately $10^{8} \mathrm{~K}$, the impurity conductivity dominates. After the accretion ends, there is a rapid cooling of the star and the conductivity of the phonons takes over.

In the last (third) phase of this evolution, we study the influence of the impurities and temperature on the magnetic field decay. We have calculated what happens with the magnetic field when the star changes its temperature (Fig. 5). In this figure, we can see that for low temperatures $T \geq 10^{4} \mathrm{~K}$, the magnetic field depends on the exact value of the impurities. For higher temperatures, $T=$ $10^{7} \mathrm{~K}$, the decay is practically independent of the precise value of $Q$. Therefore, in the last stage of evolution of the pulsar the impurities are not the most important factor for the magnetic field decay: Our study shows that the most important factor is in fact the value of the temperature, since the observed temperature of the pulsar is $\sim 10^{6} \mathrm{~K}$.

\section{DISCUSSION AND CONCLUSIONS}

We have presented the calculations of the magnetic field evolution tracking the whole history of $\sim$ Gyr black widows observed now in the ablation of the companion phase. We were able to show that after Gigayears of evolution, the magnetic field settles in a bottom field. Our calculations tentatively show that the most important factor for the temporal evolution of the $B$ is the temperature instead of the exact value of impurities. This may be a unique feature of 


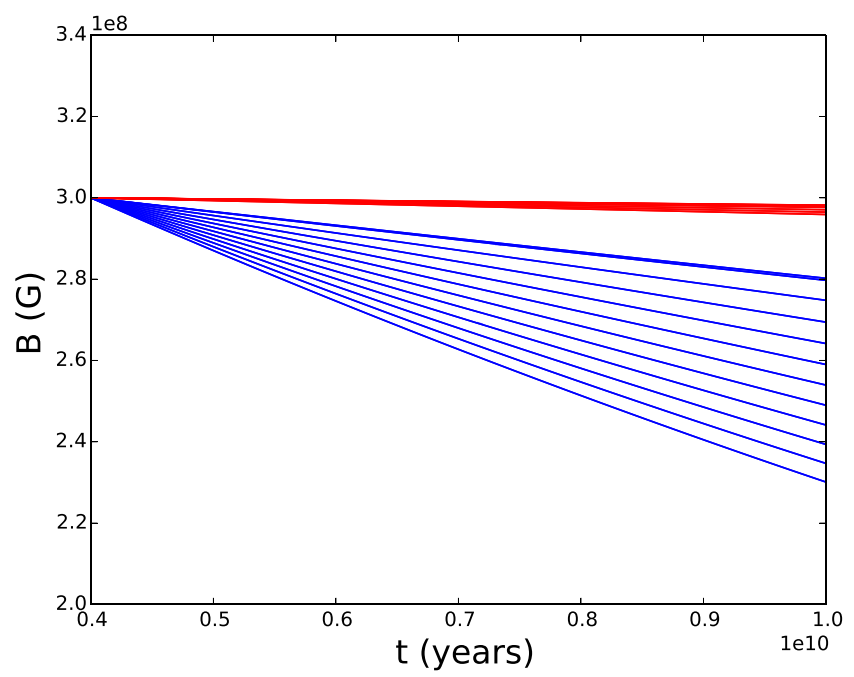

Figure 5. The magnetic field decay when we change the impurities for the temperature $T=10^{7} \mathrm{~K}$ (red lines) and $T=10^{4} \mathrm{~K}$ (blue lines).

very long-lived systems in which evolutionary considerations are different from the standard low-mass X-ray binary case.

There are three factors that influence the absolute value of the bottom field. The first one is related to the accretion process: the accretion rate and the duration of the accretion phase. The stronger and longer is the accretion, the more and faster the magnetic field decays. This would lead to a lower value of the bottom field strength. The second factor is the crustal temperature evolution. Of course the accretion rate heats up the crust, making its temperature higher, as showed by equation (5), but once the accretion stops, the cooling mechanism acts fast. In the end, it is the temperature regime that would tell what keeps the bottom field in its value: If the superficial temperature is too low, the temperature itself would not keep the field; the impurities would. The third factor is the value of the initial magnetic field. For a fixed configuration that leads to $g(x, t)$, the evolution scales with $\left|\boldsymbol{B}_{0}\right|$ and thus, to reach what is observed in black widow systems, the inferred value is $\left|\boldsymbol{B}_{0}\right| \sim 10^{13} \mathrm{G}$. So, in the configuration fixed here, a different value for $\left|\boldsymbol{B}_{0}\right|$ would lead to a different value of the bottom field. However, it must be emphasized that the field decay behaviour, given by $g(x, t)$, does not depend on the initial value of $\left|\boldsymbol{B}_{0}\right|$ in our work. This behaviour depends quite strongly upon the depth the field penetrates into the crust, which is, in turn, given by our chosen value of depth parameter $x_{0}$.

We fixed $x_{0}=1.039$, and therefore the $g$ function behaviour remained fixed (as, for example, Urpin \& Muslimov 1992). Within this approach with fixed $x_{0}$, the choice of another value for $\left|\boldsymbol{B}_{0}\right|$ will shift the curve upwards or downwards without changing the shape of the $g$ curve, i.e. the behaviour of the field decay (see, for a discussion about this point Geppert \& Urpin 1994; Urpin \& Muslimov 1992).

A remark should be added to better explain our conclusions at this point. We assert that it is the temperature that keeps the field because the observed temperature of black widows is $T \gtrsim 10^{6} \mathrm{~K}$, safely in the regime where it is the temperature that keeps the value of the bottom field (see Figs 4 and 5).

Now, a last issue should be addressed, the Hall effect. As shown in Figs 1 and 2, the Hall effect is at most of the same order of the Ohmic decay and it does not affect appreciably the magnetic field decay. Thus, the 'error' assumed in neglecting the Hall effect in our approach is as if we 'doubled' the Ohmic decay. So, in the end, as explicitly shown in Fig. 2, the Hall effect can be safely neglected.
Before moving on, some words should be dedicated to why we made our calculations using a neutron star with $M=1.33 \mathrm{M}_{\odot}$ instead of $M=1.6-1.8 \mathrm{M}_{\odot}$ measured for millisecond pulsars. From Valentim, Rangel \& Horvath (2011), we know that the mass distribution of neutron stars is double peaked at $M=1.37$ and $1.73 \mathrm{M}_{\odot}$. Because of the initial mass function, we know that most of the neutron stars are born from the less massive and more abundant progenitors (recall that here the less massive are progenitor with $M$ $\gtrsim 12 \mathrm{M}_{\odot}$ ). So we assumed that at the beginning of the evolution the mass of the neutron star came from such progenitor. As the accretion proceeds, the neutron star mass increases, but there are estimations that the accretions of $M=0.1 \mathrm{M}_{\odot}$ would cause a change of only $M=0.004 \mathrm{M}_{\odot}$ in the crustal mass, the rest being pushed into the core. Then, in the end, the mass of the neutron star increases with time, but most of this mass is concentrated in the deep regions of the star, with the crustal mass and its density profiles almost unchanged. Then, we chose without loss of generality the most common mass for neutron stars, the differences in computations being negligible.

However, and assuming that the schematic $B$ evolution is correct, there are still important questions to solve the whole evolutionary picture. We address the issue of the temperature evolution first.

We assume (Benvenuto et al. 2012) that the last phase lasts approximately 5-6 Gyr, and for this time-scale, the simplest cooling model tell us that the temperature of the star should be very low, an expectation which is not observed. On the contrary, observations tell us that the temperature of the pulsars in the redback phase is approximately $8 \times 10^{6} \mathrm{~K}$ and for the black widow phase the temperature is still $2-5 \times 10^{6} \mathrm{~K}$ (Gentile et al. 2014). Therefore, we may consider that within the time-scale of this phase (5-6 Gyr), the temperature is approximately constant. In short, the pulsars do not cool as the models predict.

To further quantify this statement, we address the cooling process of these pulsars. The evolution of the temperature (neglecting General Relativity and complications) is

$C_{v} \frac{\mathrm{d} T}{\mathrm{~d} t}=-L_{v}$,

where $C_{v}$ is the thermal capacity, $T$ is the temperature of the star, $t$ is the time evolution, and $L_{v}$ is the neutrino luminosity. Considering the URCA process as the main effect that cools the star, two types of cooling are expected: slow neutrino cooling and fast neutrino cooling (Page, Geppert \& Weber 2006).

The slow neutrino cooling occurs when we have a neutron star with a mass of approximately $1.5 \mathrm{M}_{\odot}$, in which the Modified URCA and Bremsstrahlung process dominate. For more massive stars (around $2.5 \mathrm{M}_{\odot}$ ) and higher densities, we have the fast neutrino cooling driven by Direct URCA and Page et al. (2006).

In what follows, we assume $C_{v}=C T$, which results in

$L_{v}^{\text {slow }}=N^{s} T^{8} \quad$ and $\quad L_{v}^{\text {fast }}=N^{f} T^{6}$,

where $C=10^{30} \mathrm{erg} \mathrm{K}^{-2}$.

Knowing the cooling law of the star and the luminosity $L_{v}$, we can find the constants $N^{s}$ and $N^{f}$. Since a typical redback age is approximately $4 \mathrm{Gyr}$ and its temperature $8 \times 10^{6} \mathrm{~K}$, while a black widow age is approximately $10 \mathrm{Gyr}$ and its temperature $2 \times 10^{6} \mathrm{~K}$, we use the equations 13 and 14 to find the constants $N^{s}=9.93 \times 10^{-41}$ and $N^{f}=3.08 \times 10^{-23}$.

Comparing this results with the values from the paper (Page et al. 2006) for this two process $\left(N^{s}=10^{-32}\right.$ and $\left.N^{f}=10^{-9}\right)$, we conclude that these two values are lower than their theoretically expected ones by several orders of magnitude. In other words, according to the current models, the neutron stars should have a much lower 
temperatures than the observed ones. One possible solution is to call for some agent that heats up the star to compensate the neutrino cooling, recalling that there is no accretion in this third phase.

Using equation (13) again and adding a heat luminosity $\left(L_{\text {heat }}\right)$, we can estimate the value of this putative luminosity to obtain the temperatures observed in black widow pulsars,

$C_{v} \frac{\mathrm{d} T}{\mathrm{~d} t}=-L_{v}+L_{\text {heat }}$.

Considering that the evolution time-scale from redbacks to black widow pulsars is quite long and that the temperature variation is very small, we can set $\frac{\mathrm{d} T}{\mathrm{~d} t} \approx 0$ and conclude that

$L_{\text {heat }} \approx L_{v}\left(T_{\text {redbacks }}\right)$.

Knowing that $T_{\text {redbacks }} \approx 8 \times 10^{6} \mathrm{~K}$, we found that $L_{\text {heat }} \approx$ $1.68 \times 10^{23} \mathrm{erg} \mathrm{s}^{-1}$ for the slow neutrino cooling process and $L_{\text {heat }} \approx$ $2.62 \times 10^{32} \mathrm{erg} \mathrm{s}^{-1}$ for the fast neutrino cooling process. These are the order-of-magnitude luminosities of some internal mechanism that heats up the pulsar.

It could be that the Ohmic dissipation in the third evolutionary phase heats up the star, keeping the high temperature. In order to further check this, we fitted a function $B(t)$ to the field decay part in the third phase and estimated the energy generated by this process by $E_{\text {diss }} \sim \frac{V_{\text {crust }} B \dot{B}}{4 \pi} \sim-3.5 \times 10^{35} \mathrm{erg}$. This would give an average heating rate of $\sim 5.5 \times 10^{20} \mathrm{erg} \mathrm{s}^{-1}$, three orders of magnitude less than the minimum $L_{\text {heat }}$ calculated above, causing a negligible change in the temperature even along 2 Gyr. Recall also that the cooling mechanism acts fast.

Althoughsome proposals have been advanced in connection with the problem of high temperatures inferred for old pulsars (Gentile et al. 2014), the definite long ages attributed to redbacks-black widows through evolutionary models allow a new twist on this problem extending the discrepancy to $\sim$ several Gyr old objects.

In summary, in this paper, we have studied the evolution of the magnetic field of redback-black widow pulsars. We have considered the two main ingredients of field decay: the Ohmic diffusion and the advection caused by the accretion of matter. After performing specific calculations, we conclude that the magnetic field does not decay below a bottom field $\left(2 \times 10^{8} \mathrm{G}\right)$, even within the very long evolution time inferred for these systems from evolution calculations (Benvenuto et al. 2012), provided they start with a $10^{13} G$ value at birth. In fact, these results extend the idea that magnetic fields do not decay below a bottom value for objects that are $\sim 100$ times older than ordinary field millisecond pulsars, and we consider this the main result of this study.

The constancy of the magnetic field after reaching the bottom field value is driven by the pulsar temperature, which also seems to remain quite constant, indicating that some mechanism in the pulsar can be heating up this star. Ultimately, this constancy of the temperature is entangled with the absence of decay of the magnetic field for very long times.

\section{ACKNOWLEDGEMENTS}

CM acknowledges the support from FAPESP (process 2013/044428) and from CAPES. MGBdA acknowledges the support from
FAPESP funding agency (process 2011/23996-9, 2015/20553-0). MGBdA and JEH acknowledge FAPESP Thematic Project in Compact Objects (process 2013/26258-4). JEH acknowledges FAPESP and CNPq for the support. The authors also thank Dr. Luis H S Kadowaki for his useful comments on the code we used. Much of this work could not have been possible without his expertise.

\section{REFERENCES}

Aguilera D. N., Pons J. A., Miralles J. A., 2008, A\&A, 486, 255

Baym G., Pethick C., Sutherland P., 1971, ApJ, 170, 299

Becker W., 2009, Neutron Stars and Pulsars. Springer, Berlin, Heidelberg

Benvenuto O. G., De Vito M. A., Horvath J. E., 2012, ApJ, 753, L33

Benvenuto O. G., De Vito M. A., Horvath J. E., 2014, ApJ, 786, L7

Cumming A., 2002, MNRAS, 333, 589

Cumming A., Arras P., Zweibel E., 2004, ApJ, 609, 999

De Vito M. A., Benvenuto O. G., 2010, MNRAS, 401, 2552

Elfritz J. G., Pons J. A., Rea N., Glampedakis K., Viganò D., 2016, MNRAS, 456,4461

García F., Aguilera D. N., Romero G. E., 2014, A\&A, 565, A122

Gentile P. A. et al., 2014, ApJ, 783, 69

Geppert U., Urpin V., 1994, MNRAS, 271

Glendenning N., 1996, Compact Stars. Springer, New York

Igoshev A. P., Popov S. B., 2015, Astron. Nachr., 336, 831

Itoh N., Kohyama Y., Matsumoto N., Seki M., 1984, ApJ, 285, 758

Konar S., 1997, PhD thesis, JAP

Konar S., 2017, J. Astrophys. Astron., 38, 47

Miralda-Escude J., Paczynski B., Haensel P., 1990, ApJ, 362, 572

Negele J. W., Vautherin D., 1973, Nucl. Phys. A, 207, 298

Page D., Geppert U., Weber F., 2006, Nucl. Phys. A, 777, 497

Payne D. J. B., Melatos A., 2004, MNRAS, 351, 569

Roggero A., Reddy S., 2016, Phys. Rev. C, 94, 015803

Savonije G. J., 1978, A\&A, 62, 317

Saz Parkinson P., Belfiore A., Razzano M., 2014, High Energy Astrophysics Division 14th Meeting. Am. Astron. Soc., Chicago, p. 114.03

Sengupta S., 1998, ApJ, 501, 792

Shibazaki N., Murakami T., Shaham J., Nomoto K., 1989, Nature, 342, 656

Slattery W. L., Doolen G. D., Dewitt H. E., 1982, Phys. Rev. A, 26, 2255

Urpin V. A., Muslimov A. G., 1992, MNRAS, 256, 261

Valentim R., Rangel E., Horvath J. E., 2011, MNRAS, 414, 1427

van den Heuvel E. P. J. V. D., 2011, Compact Stars and the Evolution of Binary Systems. World Scientific Press, Singapore, p. 55

Viganò D., Pons J. A., Miralles J. A., Rea N., 2015, in Cenarro A. J., Figueras F., Hernández-Monteagudo C., Trujillo Bueno J., Valdivielso L., eds, Highlights of Spanish Astrophysics VIII. XI Scientific Meeting of the Spanish Astron. Soc., Teruel, p. 90

Wang J., Zhang C. M., Zhao Y. H., Kojima Y., Yin H. X., Song L. M., 2011, A\&A, 526, A88

Wiringa R. B., Fiks V., Fabrocini A., 1988, Phys. Rev. C, 38, 1010

Zdunik J. L., Haensel P., Paczynski B., Miralda-Escude J., 1992, ApJ, 384, 129

Zhang C. M., 2013, in van Leeuwen J., ed., Proc. IAU Symp. 291, Neutron Stars and Pulsars: Challenges and Opportunities after 80 years. Beijing, China, p. 583

Zhang C. M., Kojima Y., 2006, MNRAS, 366, 137

This paper has been typeset from a $\mathrm{T}_{\mathrm{E}} \mathrm{X} / \mathrm{LAT}_{\mathrm{E}} \mathrm{X}$ file prepared by the author. 